Document downloaded from:

http://hdl.handle.net/10251/64594

This paper must be cited as:

Herrera-Tapia, J.; Manzoni, P.; Hernández-Orallo, E.; Tavares De Araujo Cesariny Calafate, CM.; Cano Escribá, JC. (2015). Power consumption evaluation in vehicular opportunistic networks. 12th IEEE Consumer Communications and Networking Conference (CCNC 2015). IEEE. doi:10.1109/CCNC.2015.7158100.

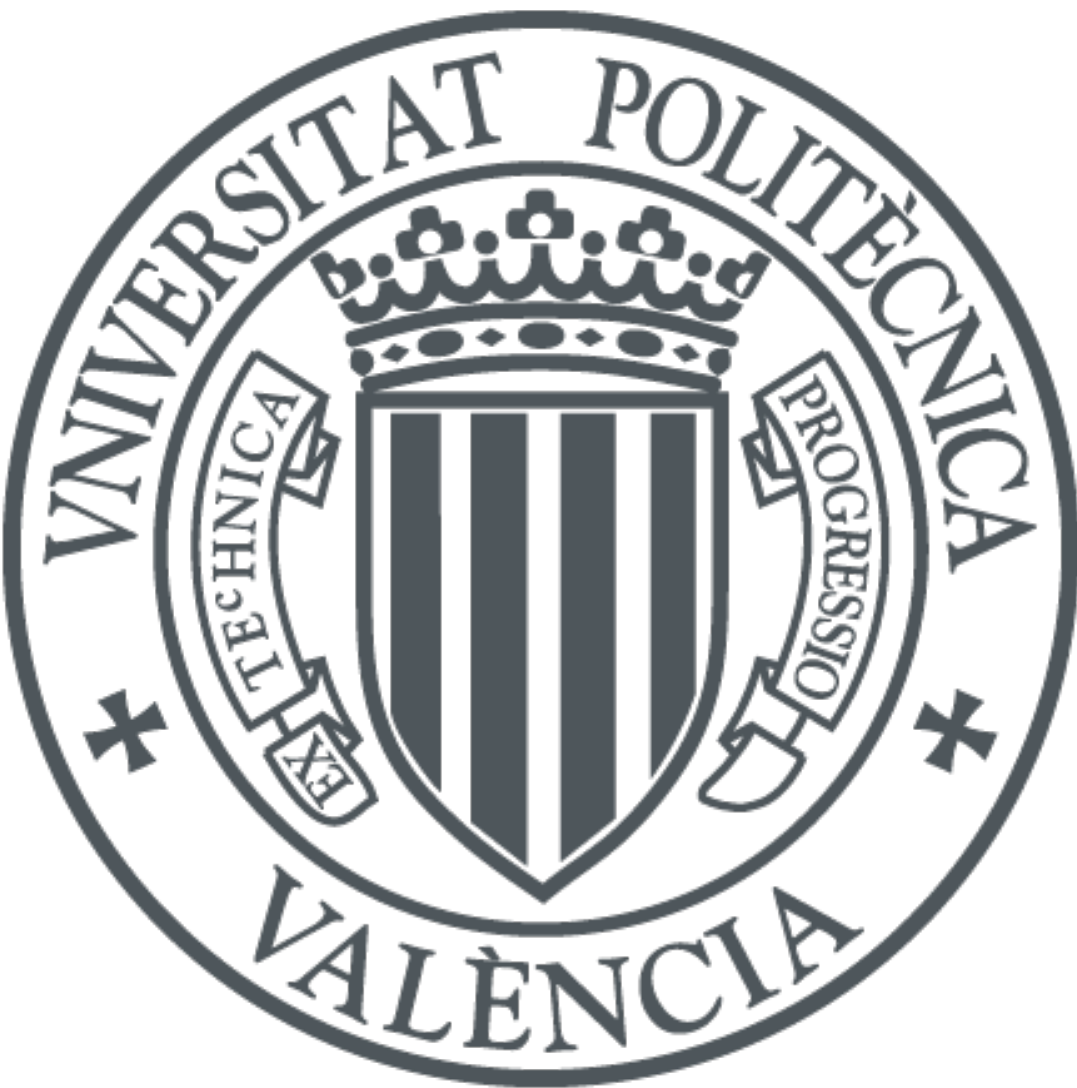

The final publication is available at

http://dx.doi.org/10.1109/CCNC.2015.7158100

Copyright IEEE

Additional Information

(C) 2015 IEEE. Personal use of this material is permitted. Permission from IEEE must be obtained for all other uses, in any current or future media, including reprinting/republishing this material for advertising or promotional purposes, creating new collective works, for resale or redistribution to servers or lists, or reuse of any copyrighted component of this work in other works. 


\title{
Power Consumption Evaluation in Vehicular Opportunistic Networks
}

\author{
Jorge Herrera-Tapia, Pietro Manzoni, Enrique Hernández-Orallo, \\ Carlos T. Calafate, Juan-Carlos Cano \\ Department of Computing Engineering \\ Universitat Politècnica de València, Valencia, Spain. \\ Email: jorherta@doctor.upv.es, \{pmanzoni, ehernandez, calafate, jucano\}@disca.upv.es
}

\begin{abstract}
The duration of batteries is a key factor that must be considered in the design and deployment of applications and services on mobile devices. The users of this technology are not aware of the energetic impact of the applications executing in their devices, especially if these applications present an adaptive behavior.

The goal of this paper is to study the power consumption associated to an opportunistic communication application. This will be achieved by analyzing the different operation stages of an application running on mobile and vehicular devices, using basic statistical analysis calculations on data collected by a logging application. Furthermore, this will allow analysing the impact of this kind of applications regarding battery performance, providing useful information to users and developers.
\end{abstract}

The experiments were executed in an opportunistic network environment, using a floating content approach as a mechanism for information distribution in a geographically delimited zone.

\section{INTRODUCTION}

Opportunistic Wireless networks [1], [2] allow the establishment of communication between mobile devices in environments where no wireless infrastructure is available. Sending and receiving information only depends on mobility, and the opportunity of contacting other devices, as long as they are willing to collaborate.

Opportunistic networks, due to their particular behavior are being subject to many investigations. In [3] and [4] the authors analyzed the performance of these networks from a temporal and spatial perspective by focusing on the inter-contact times between mobile devices.

One of the main problems of mobile devices is the power consumption in the data transmission process, from device mobile discovery to data delivery. In [5] the authors present a quantitative research to save energy through delayed transmission using $\mathrm{Wi}-\mathrm{Fi}$ and $3 \mathrm{G}$ technology. They suggest the activation and de-activation of networking interfaces in mobile devices according to specific places. They demonstrate that by delaying the transmission 100 seconds, the achievable throughput and energy gain is less than 3\%, whereas with 1 hour or longer deadlines, traffic and energy saving gains increase by $29 \%$ and $20 \%$, respectively. Furthermore, they propose a simulator based on a distribution model and a theoretical framework that enables analytical studies of the average performance of offloading.

The authors of [6], [7] present practical and in-depth studies to identify and understand the common factors related to power consumption. They describe the impact of the hardware components utilization on different types of applications, highlighting what, where and how is battery energy being spent. This must be considered when developing applications, especially when applications are running and searching for a particular service offered by another application.

The authors of [8] suggest that applications must be designed in a modular structure to optimize energy resources, and so the required modules would be loaded on demand. Instead, the authors of [9] propose changes at routing protocol levels according to the priority of forwarding nodes, but they discard messages that were sent on minor power nodes. This approach involves extra mechanisms to control reception and transmission. There are other proposals such as [10] that are based on simulations and real traces. In a similar way, they propose communication protocol changes, arguing their effectiveness and low energy properties.

The studies mentioned above provided an overview of recent research related to power consumption on opportunistic networking, and the trend towards the development of models and applications able to exploit the particularities of these wireless networks.

In order to make a realistic analysis, we proceeded to study the different operating stages of an opportunistic network application on a mobile device. This allowed us to obtain an approximation of energy consumption based on the difference of the electrical values of these periods.

The data for the analysis were obtained from a scenario of opportunistic networks using Liberouter under the Floating Content paradigm and as a client device we used an Android smartphone. Liberouter [11] is a result of the research project SCAMPI [12] founded by the European Union. The SCAMPI platform is based on activities of the Delay-Tolerant Networking Research Group (DTNRG) of the Internet Research Task Force (IRTF).

This paper is organized as follows: a brief characterization of opportunistic networking is provided in Section 2. A description of the experiments and the evaluation procedure are presented in Sections 3 and 4, respectively. Finally, in Section 5 we present the conclusions and refer to future work.

\section{Opportunistic Networking OVERVIEW}

Opportunistic communication is characterized by the intermittent and short duration of contacts between pairs of mobile 


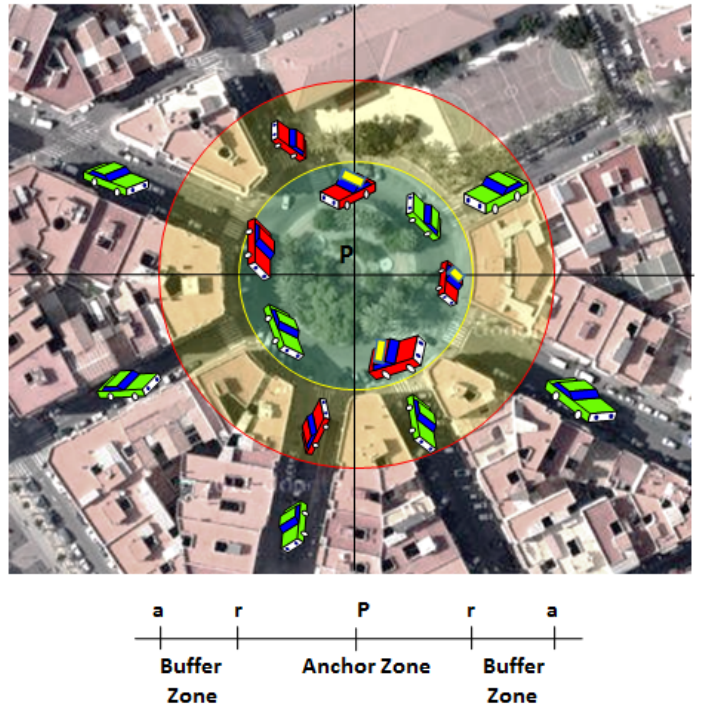

Fig. 1: Floating Content scenario.

devices. This model is considered for environments where no topology or wireless infrastructure is available for data transmission. Furthermore, it is a challenge for researchers designing and implementing protocols and applications for these environments.

Some authors [13] refer to opportunistic networks as a subclass of DTN [14] (Delay Tolerant Networks). This model is being promoted by the Internet Research Task Force group. We can find its specifications in http://www.dtnrg.org. With opportunistic networking the concept of routing protocols able to store and carry the information between mobile devices also emerged. The behavior of these networks is epidemic, and the authors of [15], [16] evaluate the performance of information dissemination.

Based on this data transmission pattern between mobile devices, authors like [17], [18] analyze the impact of human mobility on opportunistic encounters. In this context, we found definitions of Social Distance [19], [20] that assess the human mobility of employees in a work environment according their interests. These results allowed improving the routing models and proposing new solutions.

An interesting model for opportunistic communications is Floating Content [21]. In this model, sharing and distribution of information is performed in a limited geographically area called anchor zone. An anchor zone is a geographical location where mobile devices (such as vehicles) can swap contents in a floating approach, as shown in Figure 1. Floating content performance is based on three parameters of the anchor zone $(P, r, a)[22]$ where $P$ is defined as the central point of the anchor zone (this zone is where message exchanging should reach the maximum level). The values of $P$ could be set using GPS or another localization system that is currently available in most smartphones.

The central circle with radius $(r)$ represents the anchor zone, where some mobile devices can post content, while other can accept and store data copies according their interests, and replicate information if is necessary. This occurs while mobile users are inside this zone. The floating content is also parameterized with a certain lifetime to limit its availability within user devices.

When users are leaving the anchor zone and entering into the buffer zone defined by radius (a), the message replication probability decreases since, as mobile devices move away from $P$, the chances that data is erased increases.

Figure 1 also shows a realistic location of a city, where red vehicles represent mobile users with information items (yellow box) to be shared within an anchor zone. Red vehicles could be users interested in getting and storing data items and likely replicate copies, and green vehicles are normal users not interested in information.

\section{DESCRIPTION OF EXPERIMENTS}

This section describes the experimental infrastructure and the procedure used to perform power consumption measurements. First, we present the hardware and networking scheme based on Wi-Fi technology. Second, the measurement methodology is explained. It is organized in phases depending on the sequential activation of opportunistic application functionalities, since every stage has a different power consumption.

The data were captured using a Java-logging application, which was developed specifically to this purpose. Data processing was carried out of the mobile device to avoid increasing the extra power consumption.

\section{A. Testbed}

Figure 2 shows the hardware and software elements used in the experiment to collect data, organized in two groups:

1) Liberouter [23] is a framework designed to provide opportunistic communications networking. Its operation is based on the Floating Content approach [24]. This networking system consists of a SCAMPI routing platform running a Linux on Raspberry $\mathrm{Pi}$ [25] hardware with an USB Wi-Fi dongle $(802.11 \mathrm{~b} / \mathrm{g} / \mathrm{h}$

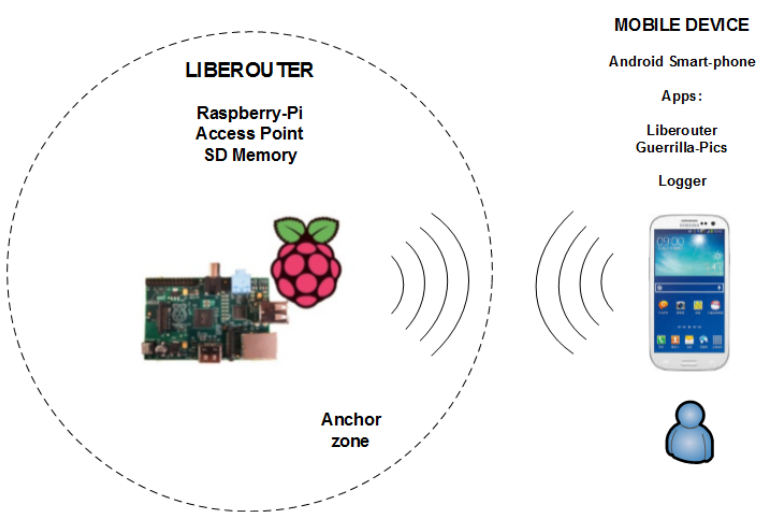

Fig. 2: Devices and tools. 

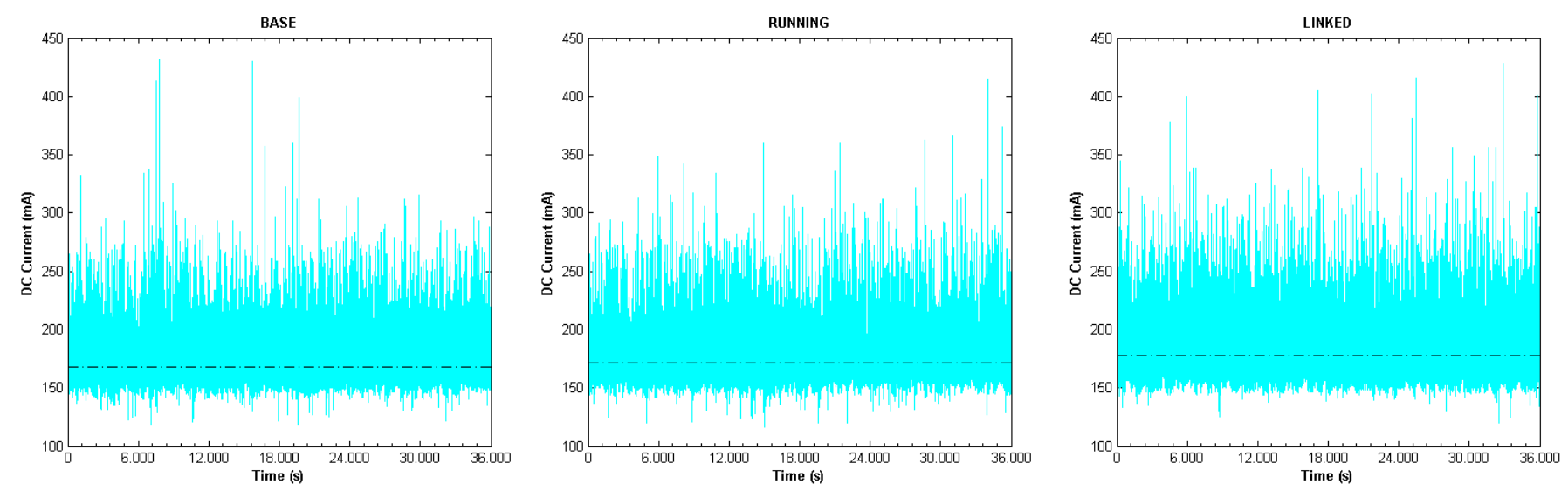

Fig. 3: Current consumption over time for different operational states.

standard). The deployment of Liberouter is easy and fast; the users just have to install the image in the Raspberry Pi SD card. After turning it On, an open wireless network called LIBEROUTER appears, allowing mobile devices to connect to this access point. Raspberry $\mathrm{Pi}$ is a cheap device that allows connecting a wide variety of hardware in its different ports. These features are the main reason to use this type of communication system in our testbed environment.

2) A mobile device composed of a Samsung Smartphone Galaxy SIII NEO running the Android 4.3 operating system. The installed applications are the following: SCAMPI router application (LibeRouter), GuerrillaPics photo sharing app and Guerrilla Tags messaging app. Furthermore, there is a logging application developed just to collect power consumption data every 0.1 seconds and transmit that information. Table I shows the structure of these records. The mathematical calculations and conversions were done externally.

\section{B. Measurement Methodology}

In order to measure the power consumption of Liberouter client apps, the smartphone just powered the Wi-Fi interface, while the GPS and other Cellular interfaces were powered Off. The logging application acquires data directly from virtual file "/sys/class/power_supply/battery". In this folder we can find text files with updated values of voltage, current and battery state, among others.

We addressed the measurements in three sequential operation stages in the mobile device: a) Base, b) Running and c)

TABLE I: Logs Structure

\begin{tabular}{c||c||c||c||c||c}
\hline No. & Time Stamp & Voltage (V) & Current $(\mathbf{m A})$ & Bytes Tx & Bytes Rx \\
\hline \hline 1 & $19: 14: 29$ & 4.25 & 258.74 & 38 & 48 \\
\hline 2 & $19: 14: 30$ & 4.15 & 298.74 & 150 & 345 \\
\hline 3 & $19: 14: 30$ & 4.26 & 198.44 & 173 & 11 \\
\hline$\ldots$ & $\ldots$ & $\ldots$ & $\ldots$ & $\ldots$ & $\ldots$ \\
\hline
\end{tabular}

Linked. The data capture lasted 1 hour in each phase, with a sampling frequency of 0.1 seconds, thereby generating 36,000 records every time. Below we describe the different phases analysed:

1) In the Base stage, we consider the regular functionality of the smartphone, without any app providing opportunistic networking. Thus, the mobile device has only the basic programs installed.

2) The Running stage takes place when the LibeRouter app is running in background without connection to any opportunistic wireless network.

3) The Linked phase takes place when the mobile device is within an anchor zone connected to the Liberouter Access Point, and their protocols are interchanging information every 10 seconds, related to the listening process.

Figure 3 shows a graphical comparison of the stages: Base, Running and Linked. We can see the density of the energy consumption according to the mobile device operation. Numerical values are shown in Table II.

Table II shows the average of DC current level on every stage. We used these values to get the DC current of the LibeRouter app computing the difference between the mean values for the Running and Base phases.

$$
\begin{aligned}
C_{\text {Liberouter }}= & C_{\text {SRunning }}-C_{\text {SBase }} \\
& 171.18 m A-168.02 m A \\
& 3.16 m A \\
C_{\text {Linked }}= & C_{\text {SLinked }}-C_{\text {SRunning }} \\
& 177.34 m A-171.18 m A \\
& 6.16 m A
\end{aligned}
$$

According to these results, we can summarize this first section with the following premises:

- The current consumption of LibeRouter is $3.16 \mathrm{~mA}$, without establishing any type of contact, as shown in Eq. 1. 
TABLE II: Power Consumption

\begin{tabular}{c||c||c||c}
\hline No. & Stages & Current $(\mathbf{m A})$ & Power $(\mathbf{m W})$ \\
\hline \hline 1 & $S_{\text {Base }}$ & 168.02 & 638.48 \\
\hline 2 & $S_{\text {Running }}$ & 171.18 & 650.47 \\
\hline 3 & $S_{\text {Linked }}$ & 177.34 & 673.88 \\
\hline
\end{tabular}

- When a mobile device is running the LibeRouter app, and it enters into anchor zone, the consumption increases by $6.16 \mathrm{~mA}$ (see Eq. 2) due to information exchanging of communication protocols, (in this case TCP). Then, the total consumption is $9.32 \mathrm{~mA}$ when LibeRouter is running into the anchor zone.

\section{EVAluATion}

Now, we conduct an evaluation based on the previous results, in order to calculate the power consumption between the different stages of opportunistic networking, and the influence on the mobile device battery.

\section{A. Power consumption during transmission}

In order to calculate the power, we multiplied each record of the collected data by the nominal battery voltage of 3.8 volts, and obtained the average value. We can see in Figure 4 the influence of the consumed current by the application. Numerical values are shown in Table II.

According to these results, the power difference is proportionally the same as for the DC current on every stage: the power consumption of Liberouter app client is $P_{\text {Running }}=$ $12 \mathrm{~mW}$, and when the mobile device is in the anchor zone it grows to $P_{\text {Linked }}=23.41 \mathrm{~mW}$. We can note that the increase is about $200 \%$, since the listening process requires more electrical resources. This factor is important to remember during the design of services for mobile phones.

While a mobile device is in the anchor zone (Linked), it receives an average of 396 Bytes and sends 290 Bytes every 1 second (this data just belongs to protocol control information). The volume of this information depends on the frequency of emitted beacons.

Once we estimated the power of the LibeRouter application in a mobile device, we addressed the same evaluations to know the power consumption when data is transmitted; then we executed the Guerrilla-Pics (Liberouter app), to send one image file of 96kBytes, and we got the following values:

- The current consumption is $C_{\text {file }}=40.25 \mathrm{~mA}$

- The power consumption is $152.95 \mathrm{~mW}$.

- $\quad$ The average time to send $96 k$ Bytes is $58 \mathrm{~ms}$.

According to these values, the energy consumption to send 96kBytes is 9 mJules.

\section{B. Impact on the battery performance}

Finally, we addressed an approximation of the impact of power consumption over time. Then, taking into account the consumption for every phase and the number of transmitted

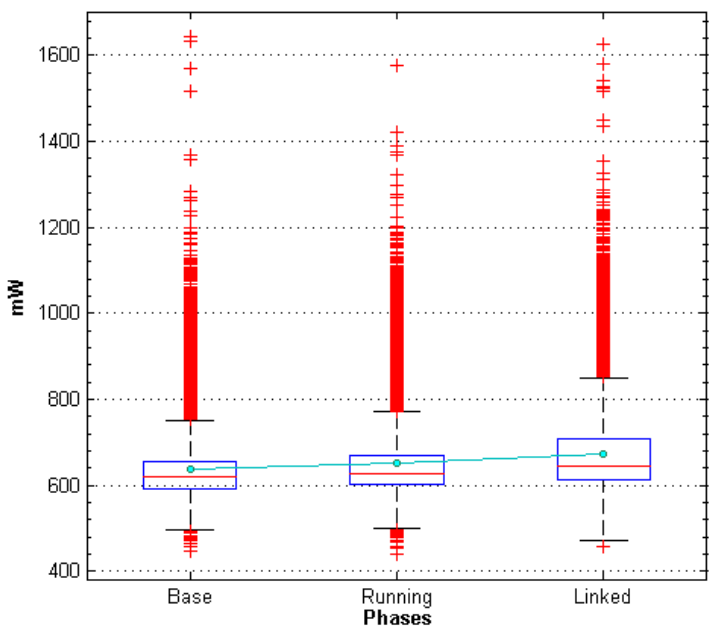

Fig. 4: Power consumption by stages.

files, the impact on the battery time is calculated using the following expression:

$$
t_{i}=\frac{Q}{C_{\text {App }}+C_{\text {Linked }} \cdot t_{a}+k \cdot C_{\text {file }} \cdot t_{f}}
$$

where $Q$ is the battery capacity (in this specific case $2100 m A h$ ); $C_{A p p}=C_{S B a s e}+C_{\text {Liberouter }}, C_{\text {Linked }}$ is the power consumption, $t_{a}$ is the time spent by the mobile device within an anchor zone, $k$ is the number of sent files, and $t_{f}$ is the time required to send a file. We analyzed two scenarios to explain the impact of the power consumption in the different phases.

In the first scenario, the time is calculated with a fixed value $t_{a}=1$ hour and varying $k$ from 5,000 to 50,000. These transmission rates can reflect, for example, a periodic information update or video transmission. Figure 5 shows the results for this scenario, being the blue solid line the relationship between the $C_{A p p}$ consumption and battery capacity. This is the reference value to compare the impact of our target application over time.

The blue dashed line represents the behavior when the mobile device enters in an anchor zone without any data transmission, and the red lines represent the case when mobile users are transmitting. As we can, see the impact of the transmission is lower when the mobile device is linked to an anchor zone. The battery duration is no less than 10 hours.

In the second scenario, the time is calculated by changing the spent time $t_{a}$ by a mobile device within the anchor zone from 1 hour to 8 hours, and $k$ is fixed to 5,000 files. Figure 6 shows the result of this approximation. The blue solid lines have the same meaning of the previous figure, but the red lines represent the spent time by a mobile device in the anchor zone. The effect of the file transmission is not significant with respect to the contact and permanence of a mobile user in the anchor zone. In this case, the battery time decreases to near to 9 hours. 


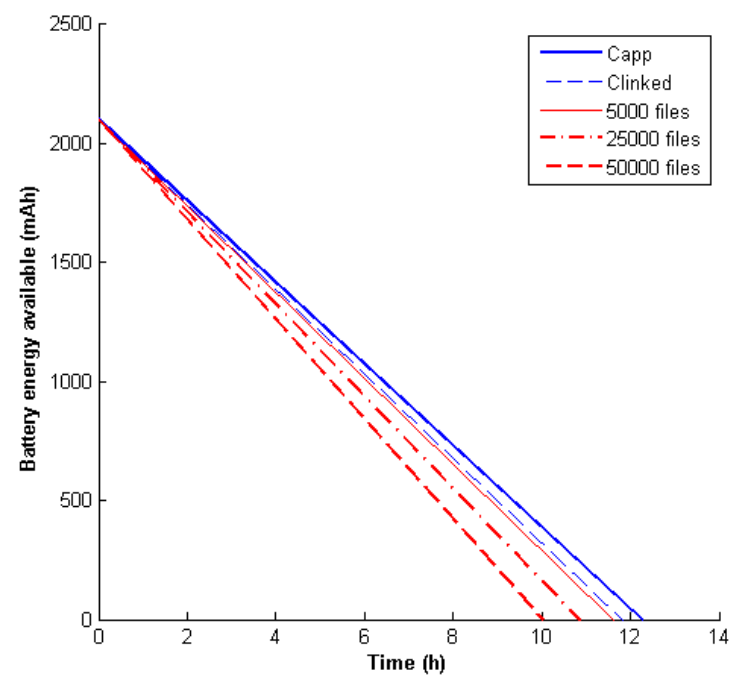

Fig. 5: Impact of data transmission.

Summarizing, we can see that the impact of opportunistic networking applications when the mobile device sends information, is lower than merely remaining within an anchor zone. The Linked phase has the greatest power consumption, when the devices exchange information. Finally, the transmission data phase consumes less power because this action is intermittent, taking place only when there is an opportunity to establish a connection with another wireless device.

\section{CONCLUSIONS AND FUTURE WORK}

The main objective of this paper was to perform power consumption measurements to study the impact of an application offering opportunistic networking. Based on the results, our evaluation showed that the power consumption in

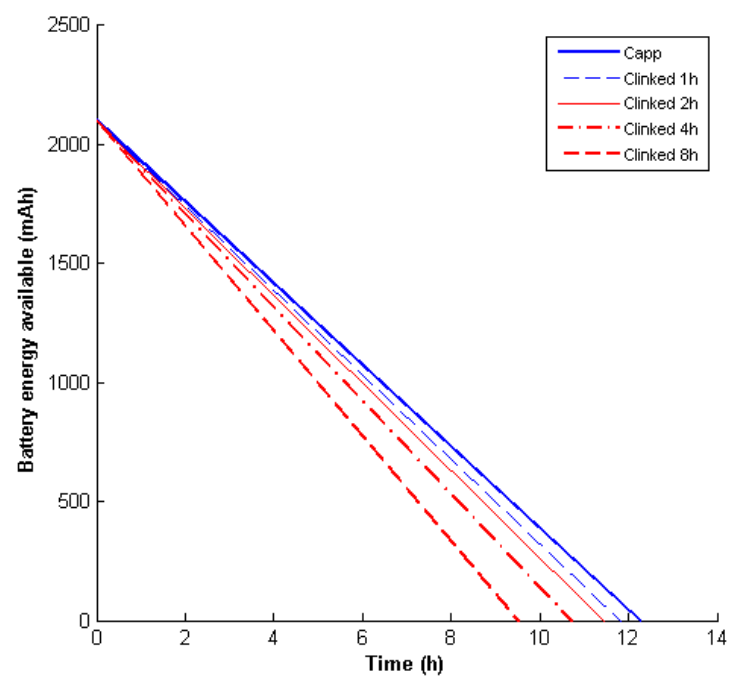

Fig. 6: Impact of the total Linked Time. an opportunistic network is moderately low, making feasible and recommendable the deployment of applications of this communications environment. Furthermore, we conclude that, in opportunistic networking, the impact on mobile device batteries is greater since the application must be running permanently. An approach to reduce the battery consumption is to have the app running only when it is in an anchor zone.

Also, we can state that, when a mobile device stays in the anchor zone, the power consumption is greater than when it is sending information. This is a consequence of the periodical swapping of protocol control information, a procedure required to receive and retransmit the packets in an anchor zone. Under these circumstances the correct and adequate tuning of this process is crucial to minimize the power consumption.

On the long term, our interest in this analysis is to increase the battery life by optimizing applications without losing their functionalities. Future works are focused on the following aspects: a) exploiting sensor information in the design and implementation of energy-efficient applications, and b) encouraging the collaboration between mobile devices by using of mobile apps designed with energy efficiency constraints.

\section{ACKNOWLEDGMENT}

This work was partially supported by the Ministerio de Ciencia e Innovación, Spain, under Grant TIN201127543-C03-01 and by the Secretaría Nacional de Educación Superior, Ciencia, Tecnología e Innovación del Ecuador (SENESCYT).

\section{REFERENCES}

[1] S. Ferretti, "Shaping opportunistic networks," Computer Communications, vol. 36, pp. 481-503, 2013.

[2] C.-M. Huang, K.-c. Lan, and C.-Z. Tsai, "A Survey of Opportunistic Networks," 22nd International Conference on Advanced Information Networking and Applications, pp. 1672-1677, 2008.

[3] E. Hernández-Orallo, J.-C. Cano, C. T. Calafate, and P. Manzoni, "A representative and accurate characterization of inter-contact times in mobile opportunistic networks," Proceedings of the 16th ACM international conference on Modeling, analysis \& simulation of wireless and mobile systems - MSWiM '13, pp. 309-316, 2013.

[4] C. D. Anna-kaisa Pietiläinen, "Experimenting with Opportunistic Networking," Proceedings of International Workshop on Mobility in the Evolving Internet Architecture (MobiArch'09), 2009.

[5] Y. Y. I. R. S. C. Kyunghan Lee, Joohyun Lee, "Mobile Data Offloading : How Much Can WiFi Deliver ?," IEEE/ACM Transactions on Networking.

[6] M. Z. Abhinav Pathak, Y Charlie Hu, "Fine Grained Energy Accounting on Smartphones with Eprof," Proceedings of the ACM SIGOPS EuroSys 2012 Conference (2012), pp. 29-42, 2012.

[7] Q. Technologies Incorporated, "WHEN MOBILE APPS USE TOO MUCH POWER A Developer Guide for Android App Performances," Technologies Incorporated, Qualcomm, 2013.

[8] M. Avvenuti, P. Corsini, P. Masci, and A. Vecchio, "Opportunistic computing for wireless sensor networks ," Mobile Adhoc and Sensor Systems. MASS 2007. IEEE Internatonal Conference on, pp. 1-6, 2007.

[9] X. Mao, S. Tang, X. Xu, X.-Y. Li, and H. Ma, "Energy Efficient Opportunistic Routing in Wireless Sensor Networks," Parallel and Distributed Systems, IEEE Transactions on, pp. 1934-1942, 2011.

[10] V. Kanakaris, D. Ndzi, and D. Azzi, "Ad-hoc Networks Energy Consumption : A review of the Ad-Hoc Routing Protocols," vol. 3, pp. 162167,2010

[11] T. Karkkainen and J. Ott, "Liberouter: Towards autonomous neighborhood networking," 2014 11th Annual Conference on Wireless Ondemand Network Systems and Services (WONS), pp. 162-169, 2014. 
[12] http://www.ict scampi.eu/, "ICT-SCAMPI,"

[13] J. Niu, J. Guo, Q. Cai, N. Sadeh, and S. Guo, "Predict and Spread : an Efficient Routing Algorithm for Opportunistic Networking," Wireless Communications and Networking Conference (WCNC), 2011 IEEE, pp. 498-503, 2011.

[14] G. S. Thakur, U. Kumar, A. Helmy, and W.-J. Hsu, "On the efficacy of mobility modeling for DTN evaluation: Analysis of encounter statistics and spatio-temporal preferences," Wireless Communications and Mobile Computing Conference (IWCMC), 2011 7th International, pp. 510-515, 2011.

[15] Y. Li, X. You, S. Lei, Q. Liu, K. Sohraby, and C. Wang, "Analysis and improvement of TCP performance in opportunistic networks," Wireless Networks, vol. 18, pp. 799-810, 2012.

[16] R. Ramanathan, R. Hansen, P. Basu, R. Rosales-Hain, and R. Krishnan, "Prioritized epidemic routing for opportunistic networks," Proceedings of the 1st international MobiSys workshop on Mobile opportunistic networking - MobiOpp '07, pp. 62-66, 2007.

[17] L. Pajevic, G. Karlsson, and O. Helgason, "Epidemic Content Distribution : Empirical and Analytic Performance,” pp. 335-339, 2013.

[18] A. Chaintreau, P. Hui, J. Crowcroft, C. Diot, R. Gass, and J. Scott, "Impact of Human Mobility on Opportunistic Forwarding Algorithms,' IEEE Transactions on Mobile Computing, vol. 6, pp. 606-620, 2007.
[19] A. Förster, K. Garg, H. A. Nguyen, and S. Giordano, "On Context Awareness and Social Distance in Human Mobility Traces Categories and Subject Descriptors," pp. 5-12, 2012.

[20] S. Gaito, E. Pagani, and G. P. Rossi, "Opportunistic Forwarding in Workplaces," WOSN '09: Proceedings of the Second ACM Workshop on Online Social Networks, pp. 55-60, 2009.

[21] J. Kangasharju, "Floating Content : Information Availability in Urban Environments University of Helsinki Email : jakangas@cs.helsinki.fi Email : jo@netlab.tkk.fi University of Helsinki Email : karkulah@cs.helsinki.fi,”pp. 804-808, 2010.

[22] J. Ott, E. Hyytiä, P. Lassila, J. Kangasharju, and S. Santra, "Floating content for probabilistic information sharing," Pervasive and Mobile Computing, vol. 7, pp. 671-689, 2011.

[23] http://www.ict-scampi.eu/results/scampi liberouter/, "Liberouter."

[24] J. Ott, E. Hyytia, P. Lassila, T. Vaegs, and J. Kangasharju, "Floating content: Information sharing in urban areas," 2011 IEEE International Conference on Pervasive Computing and Communications (PerCom), pp. 136-146, 2011.

[25] http://www.raspberrypi.org/product/model b/, "Model B - Raspberry Pi.' 